\title{
Efectos de la herbivoría foliar en el crecimiento y mortalidad de plántulas de Vochysia ferruginea (Vochysiaceae) en un bosque en regeneración pos-huracán
}

\author{
Javier Ruiz*
}

\section{Recibido: febrero de 2012 / Aceptado: abril de 2012}

La herbivoría foliar puede afectar el crecimiento y la mortalidad de plántulas en bosques húmedos tropicales. Para evaluar esta aserción se establecieron experimentos en dos secciones de un bosque húmedo tropical en regeneración tras los daños causados por el huracán Juana en 1998. Los trabajos de campo fueron llevados a cabo durante el inicio de la fase de raleo de regeneración de bosque -caracterizada por condiciones bajas de iluminación a nivel del sotobosque- en cuatro parcelas permanentes $(20 \times 20 \mathrm{~m})$. Ambos sitios fueron estudiados entre febrero 1997 y agosto 1998 y re-analizados en un estudio de los efectos de la herbivoría foliar natural en la sobrevivencia y mortalidad de plántulas de V. ferruginea de febrero 2003 a febrero 2004 en dos parcelas de 6.3 ha, una parcela por sitio. En cada parcela experimental se identificaron las plántulas con altura máxima $\leq 100 \mathrm{~cm}$, se etiquetaron con un número y se anotó su altura máxima. La herbivoría natural fue cuantificada y se aplicaron dos tratamientos de herbivoría artificial (50\% y 95\%). Con el objetivo de controlar las condiciones de iluminación (sombra versus luz) en el sotobosque, se aplicó un tratamiento de anillado de árboles en el que se remueve el tejido conductivo alrededor del tronco de cualquier especie con $>10 \mathrm{~cm}$ en circunferencia en una parcela por cada sitio. Para evaluar el efecto de los tres niveles de herbivoría artificial y los dos niveles de iluminación en el crecimiento y sobrevivencia de plántulas de $V$. ferruginea se realizó un ANCOVA. El análisis muestra que en este período el efecto de los

\footnotetext{
* Centro de Investigación y Documentación de la Costa Atlántica. Bluefields Indian $\mathbb{E}$ Caribbean University, Barrio Punta Fría, Bluefields, Nicaragua.

Department of Ecology and Evolutionary Biology. The University of Michigan, Ann Arbor MI, 48109, USA. Correo electrónico: javierruizphd@gmail.com
} 
niveles de herbivoría y las condiciones de luz en sotobosque no fue estadísticamente significativo en el crecimiento y la mortalidad de plántulas cuando se incrementaron artificialmente las condiciones de luz en el sotobosque. La única variable que mostró un efecto estadísticamente significativo sobre el crecimiento fue la altura inicial de las plántulas. Esto podría deberse a que durante este periodo las condiciones de luz permanecieron altas en el sotobosque. El modelo fue altamente significativo $\left(\mathrm{R}^{2}=0.7457, \mathrm{~F}_{5,69}=50.59, \mathrm{p}=2.2 \mathrm{e}-16\right)$. Las siguientes observaciones se realizaron durante el inicio de la etapa de raleo, donde se analizó el efecto de la herbivoría natural y la presencia de arañas en el crecimiento y sobrevivencia de plántulas de $V$. ferruginea. El análisis muestra que aunque la importancia de la herbivoría foliar sobre la demografía de las plántulas es estadísticamente significativa en esta etapa, la magnitud y dirección de este efecto varió en los dos períodos de medición: febrero 2003 y febrero 2004. Este modelo fue también altamente significativo $\left(R^{2}=0.7872\right.$, $\mathrm{F}_{5,142}=105.1, \mathrm{p}=2.2 \mathrm{e}-16$ ). Se piensa que la presencia de arañas en las plántulas altera el coeficiente de herbivoría por insectos de forma no lineal en las etapas recientes de regeneración del bosque.

Palabras clave: Demografía vegetal / efectos indirectos / investigación de larga duración / Nicaragua

\section{Introducción}

El daño de la herbivoría foliar puede afectar negativamente las plántulas (Coley, 1983; Dirzo, 1987; Coley 1990) y puede ser suficientemente severo para determinar la suerte de éstas (Sullivan, 2003). La herbivoría foliar en especies de árboles tropicales se ha estimado en 10\% del daño de las hojas (Dirzo, 1987) y la mayor parte de este daño es causado por larvas de mariposas (Dirzo, 1987; Coley, 1990; Coley \& Barone, 1996). De esta forma, una reducción en la herbivoría puede resultar en tasas altas de reclutamiento en las plantas (Coley, 1983; Coley, Bryant \& Chapin, 1985; Coley, 1990; Clark \& Clark, 1985). Las tasas bajas de reclutamiento de plántulas de algunas especies podrían deberse en parte al efecto negativo de los herbívoros artrópodos sobre las plántulas (Augspurger, 1983; Augspurger, 1984; Clark \& Clark, 1984; Augspurger \& Kitajima, 1992; Hyatt et al. 2003). Por ejemplo, en un estudio de campo se ha determinado que el reclutamiento de las plántulas de Dipteryx oleifera está negativamente correlacionado con los niveles de daños de herbivoría insectívora. Aunque este tipo de daño fue $<8 \%$ del area foliar, este estrés parece haber determinado las tasas de sobrevivencia de plántulas (Clark \& Clark, 1985). Niveles similares de daño foliar han causado reducción en el crecimiento y aumento en la mortalidad de plántulas en la especie Vochysia ferruginea (Ruiz, 1999). A pesar de que los niveles bajos de herbivoría por insectos parecieran ser ubicuos en el sotobosque, se necesita más investigación para determinar cuáles interacciones son las responsables de la sobrevivencia y crecimiento de plántulas (Clark \& Clark, 1985; Ruiz, Boucher, Ruiz-Moreno \& Ingram-Flores, 2009a).

En una comunidad biológica, las especies están interconectadas directa o indirectamente por medio de interacciones entre los consumidores y los productores 
(Carpenter et al., 2001). Un aspecto relevante en el proceso del reclutamiento de plántulas son los efectos de los depredadores sobre los herbívoros, por la aparente reducción del porcentaje de herbivoría foliar que causan. En un sistema con tres niveles tróficos, como son plantas, artrópodos herbívoros y depredadores, una cadena trófica describe el efecto de los depredadores sobre la abundancia, riqueza o la estructura del nivel de los productores (Hairston, Frederick \& Lawerence, 1960; Levins, 1974; Gruner, 2004; Moreau, Elveleigh, Lucarotti \& Quiring, 2006). Por ejemplo, ciertos tipos de arañas podrían reducir la tasa de daños de los insectos herbívoros en las plántulas y de esta forma incrementar la producción primaria (Romero \& Vasconcellos-Neto, 2004). Los efectos de los niveles tróficos mencionados sobre la demografía de plántulas están frecuentemente asociados con la reducción en la abundancia de artrópodos herbívoros (Van Bael, Brawn \& Robinson, 2003; Romero \& Vasconcellos-Neto, 2004; Van Bael \& Brawn, 2005) o la reducción de la herbivoría foliar de artrópodos (Coley, Bryant \& Chapin, 1985).

Un factor limitante en la regeneración a partir de plántulas es la disponibilidad de luz proveniente de los niveles superiores -debido a que los bosques húmedos tropicales tienen un dosel cerrado- en las etapas de raleo y bosque maduro. Brokaw (1985) propone dos estrategias de adaptación vegetal a la disponibilidad de luz en un bosque húmedo. Por un lado están las especies que requieren de condiciones plenas de luz para crecer y establecerse en el dosel. Estas son generalmente conocidas como pioneras porque son las que colonizan los espacios abiertos en el sotobosque, creados cuando un árbol cae en un dosel cerrado. Estos espacios abiertos presentan condiciones plenas de luz y son conocidos como claros de luz. Para hacer uso de estas condiciones, las especies pioneras destinan la mayor parte de su energía para crecer, en su intento por establecerse en los doseles superiores del bosque, y por esto disponen de poca energía para defensas contra los insectos herbívoros. Por otro lado, las especies de bosque maduro presentan tasas de crecimiento menor, son capaces de sobrevivir en condiciones de sotobosque de bosques cerrados y, por lo general, presentan buenas defensas químicas contra los herbívoros. Sin embargo, entre los dos extremos de historias de vida se encuentra un gran número de variaciones a esta dicotomía (Brokaw, 1985).

Una importante línea de investigación, evaluada hasta cierto punto, son los cambios en las interacciones biológicas como consecuencia de fluctuaciones en la dinámica y la estructura de bosques, o cambios relacionados con el proceso de regeneración de éstos (Dirzo, 1987; Coley, 1990; Coley \& Barone, 1996). Siendo la especie en estudio, Vochysia ferruginea, la más abundante en muchos de nuestros sitios de los bosques del este de Nicaragua, se piensa que los cambios en las interacciones planta-insecto en esta especie resultarían cruciales para estos ecosistemas. Esta investigación aborda la hipótesis de trabajo que si el crecimiento y sobrevivencia de las plántulas de $V$. ferruginea es independiente a los cambios de luz y a la cantidad del daño foliar durante el proceso de regeneración de un bosque húmedo tropical, entonces esos factores no afectarán el crecimiento ni la sobrevivencia de las plántulas. Para responder a esta hipótesis se establecieron experimentos de campo donde se manipuló la intensidad de luz y el porcentaje de área foliar perdida por las plantas. Se evaluaron las respuestas de las plantas en relación a la intensidad de luz y la herbivoría foliar. Se argumenta que la situación 
donde la luz es alta y la herbivoría es baja es un escenario que podría resultar en altas tasas de sobreviviencia de V. ferruginea. Contrastando con esta noción, se dice que debido a que las especies de bosque maduro (ej. Dipteryx oleifera) colocan más energía para defenderse (químicamente) de herbívoros y poca para crecer, en presencia de altas cantidades de luz éstas crecen puesto que por lo general no son consumidas por herbívoros. También se evaluó la hipótesis de si las arañas depredadoras de insectos son inocuas en la dinámica de reclutamiento de plántula de V. ferruginea, o si éstas juegan un papel en los cambios en el nivel de herbivoría y crecimiento de las plántulas. Investigaciones previas han presentado poca evidencia acerca del papel ecológico de las arañas sobre las poblaciones de insectos herbívoros (Basset, 2001) y se conoce poco acerca del efecto que las arañas tienen sobre la dinámica del reclutamiento de plántulas.

\section{Metodología}

\subsection{Sitios de estudio}

El estudio se realizó en dos localidades dentro del área de distribución del bosque húmedo tropical del este de Nicaragua. El primer sitio de estudio, La Unión $\left(12^{\circ} 5^{\prime} \mathrm{N}\right.$ $\left.83^{\circ} 53^{\prime} \mathrm{W}\right)$, se encuentra aproximadamente a $10 \mathrm{~km}$ de la ciudad de Bluefields - la capital de la Región Autónoma del Atlántico Sur. El segundo sitio de estudio es La Bodega (11' 52' N, 83 58' W), aproximadamente a 20 km de Bluefields. Una descripción detallada y un mapa de ambos sitios fue publicada con anterioridad (Granzow de la Cerda, Zamora, Vandermeer \& Boucher, 1997).

\subsection{Especie en estudio}

V. ferruginea es una especie que puede alcanzar de 30 a 50 metros de altura y sus plántulas pueden resistir niveles altos de herbivoría foliar (Ruiz, 1999). Es la especie más abundante de los bosques post huracanados, representa el 42\% (224 de 523) de los árboles $>200 \mathrm{~mm}$ de diámetro a la altura del pecho y una densidad de 87 individuos por hectárea en el bosque del sitio La Bodega (Boucher \& Granzow de la Cerda, 2008). Según Yih, Boucher, Vandermeer \& Zamora (1991), la mayor parte de las especies resistieron los vientos sostenidos de aproximadamente 240 kilómetros por hora del huracán Juana en 1988. Sin embargo, V. ferruginea experimentó una mortalidad total de individuos adultos en las parcelas, siendo su regeneración a partir del banco de semillas y plántulas presentes en el sotobosque antes del huracán. En los últimos años, las especies se encuentran compitiendo en el dosel compacto que se formó después del huracán a unos 30 metros de altura.

\subsection{Efectos de la herbivoría artificial y los niveles de luz en el crecimiento de las plántulas}

Se procedió a establecer un experimento para evaluar el efecto de dos condiciones de luz en el sotobosque (luz y sombra) y la herbivoría foliar experimental. Los 
experimentos de campo se realizaron en un total de cuatro parcelas experimentales de 20x20m establecidas en cada sitio, dos en La Unión y dos en La Bodega, en febrero de 1997. Para la selección del área donde se establecieron las parcelas se tomó como parámetro la presencia de plántulas de $V$. ferruginea, las cuales fueron localizadas e identificadas dentro de cada parcela. Se consideró plántulas a todos aquellos individuos con altura $\leq 100 \mathrm{~cm}$ (Clark \& Clark, 1984; 1985). Cada plántula fue identificada con una placa de aluminio numerada, que fue asegurada con un alambre inoxidable alrededor del tallo. La altura máxima (distancia vertical desde el suelo del bosque hasta la posición del tejido vegetal verde más alto) y el porcentaje de herbivoría natural al inicio del experimento y al final del mismo (febrero 1997 y agosto 1998, respectivamente) fueron registrados para cada plántula. Las plántulas fueron registradas como muertas (en agosto 1998) cuando las placas fueron encontradas solas o cuando la plántula estaba completamente marchita y sin tejidos verdes.

En febrero de 1997 se realizó el primer experimento que incluyó dos tratamientos: uno de intensidad de luz y otro de herbivoría. El tratamiento de intensidad lumínica analizó dos condiciones de luz (sombra y luz). La disponibilidad de la luz en el sotobosque se favoreció mediante la técnica de anillamiento forestal, que fue aplicada en febrero de 1997. Esta técnica se aplicó a todos los árboles >10 m de altura dentro de una parcela de 20x20 m establecida en cada sitio de estudio. El anillamiento interrumpe la circulación del floema e induce a la muerte del árbol sin perturbar el sotobosque con la caída del árbol y facilita la entrada de luz en los estratos bajos.

El tratamiento de herbivoría foliar se manipuló de forma artificial de tal manera que representara un $50 \%$ y un $95 \%$ de daño en el total de área foliar de la plántula. Para realizar esta fase se utilizaron tijeras con sacabocado de forma circular y una plántula fue asignada como testigo, a la que se le midió visualmente el porcentaje de herbivoría foliar natural. Las variables dependientes en este experimento fueron las alturas de las plántulas al inicio y al final del experimento. En cada parcela se evaluaron 30 plántulas para un total 120 plántulas en las cuatro parcelas.

Los datos de campo fueron estudiados con un análisis de covarianza (ANCOVA) que se utilizó para evaluar el crecimiento en altura de todas aquellas plántulas que tenían todos sus registros completos en la hoja de datos de campo, para un total de 96 plántulas durante un periodo de un año de experimentos de campo entre 1997 y 1998. Los datos de las alturas fueron transformados con la función del arco del seno para normalizar los datos. El ANCOVA también permitió analizar el efecto de la altura inicial de los tres niveles de herbivoría (herbivoría natural $\sim 10 \%, 50 \%, 95 \%$ ) y la condición de intensidad de iluminación (sombra y luz) sobre la altura final de plantas un año después:

$$
Y_{t}=\mu+\alpha Y_{t-1}+\beta_{1} H_{a}+L_{b}+\varepsilon
$$

Donde $\mu$ es el intercepto, el cual da una idea de la media del crecimiento de cada plántula, $\alpha$ es un parámetro que relaciona la altura a ser estimada un año después $\left(\mathrm{Y}_{\mathrm{t}}\right)$ con la altura del año anterior. $\mathrm{Y}_{\mathrm{t}-1}, \beta_{1}$ representa el efecto de tres niveles de herbivoría artificial $\left(\mathrm{H}_{\mathrm{a}}\right)$ en el crecimiento en altura, L corresponde a la condición de luz o sombra introducida al modelo como una covariable de dos entradas $(1,0)$ 
y $\varepsilon$ representa al error estadístico asumido como i.i.d. (Venables \& Ripley, 2002). También se utilizaron dos análisis de Chi-Cuadrado con la corrección de Yates para evaluar el efecto de tres niveles de herbivoría sobre la mortalidad de plantas. Los tres niveles de herbivoría fueron incluidos en el análisis como las variables independientes y las variables dependientes correspondieron a las observaciones de sobreviviencia y mortalidad. El análisis se realizó para la condición de sombra y otro para la condición de plena luz.

\subsection{Efecto de la herbivoría natural sobre el crecimiento y mortalidad de plántulas}

Con el fin de evaluar estos aspectos, el estudio se realizó en una parcela permanente de 6.37 ha establecida en La Unión y otra de 6.35 ha en La Bodega. En ambos sitios las parcelas fueron divididas en sub-parcelas de 10x10 m. Las coordenadas del sitio coinciden con la localización de la esquina noreste de las parcelas. Dentro de éstas, las plántulas de V. ferruginea fueron localizadas en un área rectangular de 3 ha en cada sitio. Se anotaron la altura y el porcentaje de área foliar dañada por herbivoría natural en cada plántula de V. ferruginea dentro de la sub-parcela de 3 ha. El tamaño de la muestra evaluada fue de 298 plántulas de V. ferruginea entre febrero 2003 y febrero 2004. Con el objetivo de calcular el porcentaje de herbivoría foliar para cada plántula el área foliar consumida por herbívoros fue sumada y dividida entre el número total de hojas.

De manera adicional se observó la presencia de arañas en las hojas de las plántulas de V. ferruginea en las parcelas permanentes en febrero 2003. Muestras de especímenes de arañas fueron recolectadas fuera del área de estudio en febrero 2004 y fueron depositadas en el Museo de Historia Natural de la Universidad Centroamericana en Managua. Se llevó a cabo una descripción de la morfología de cada morfo-especie de araña (Ruiz, Boucher, Ingram-Flores \& Chaves, 2009b). La presencia o ausencia de las arañas fue anotada individualmente para cada plántula en febrero 2003 y en febrero 2004. Se utilizaron dos categorías para la localización de arañas en cada plántula de V. ferruginea: (1) Presente, cuando las arañas fueron encontradas en una plántula o cuando una tela de araña abandonada fue encontrada entre o bajo las plántulas. Por lo general, la presencia de arañas se evidenció por un individuo adulto. (2) Ausente, sin arañas ni otra evidencia de la presencia de arañas.

Un segundo ANCOVA se utilizó para evaluar el crecimiento de 149 plántulas a las que se les midió altura, porcentaje de herbivoría y presencia/ausencia de arañas en febrero 2003 y febrero 2004. Los datos de las alturas fueron transformados con la función de arco del seno para normalizar los datos. El modelo estudia el efecto de la altura inicial, de la herbivoría foliar natural ( 10\%) y la presencia de arañas sobre la altura de plantas en tiempo $t$ :

$$
Z_{t}=\mu+\alpha Z_{t-1}+\beta_{1} D_{a}+A_{b}+A_{c}+\varepsilon
$$

Donde $\mu$ es el intercepto y corresponde a la media del crecimiento de cada plántula, $\alpha$ es un parámetro que relaciona la altura a ser estimada un año después $\left(Z_{t}\right)$ con la altura del año anterior $Z_{t-1}, \beta_{1}$ es el efecto de tres niveles de herbivoría 
$\left(D_{\mathrm{a}}\right)$ en el crecimiento en altura, A corresponde a la presencia o ausencia de arañas, una variable introducida al modelo como una covariable de dos entradas $(1,0)$ y $\varepsilon$ representa el error estadístico asumido como i.i.d.

Se utilizó una prueba T de Student con el objetivo de determinar si existe diferencia estadística en el nivel de daño que sufren las plantas que sobreviven en comparación con las que mueren en el siguiente año. La condición fue introducida como una variable categórica de dos niveles (vivo o muerto) y una variable numérica continua (el porcentaje de herbivoría foliar natural).

Se graficó la media y el error estándar de herbivoría foliar natural en t-1 contra la media y el error estándar de herbivoría en tiempo t para cada una de las condiciones (vivo o muerto). En otro gráfico, una línea de 45 grados que parte del origen, fue dibujada a la gráfica con los datos de herbivoría en tiempo t. La herbivoría en tiempo inicial $t-1$ como variable dependiente (en el eje y) y la distancia del individuo congénere más cercano durante el período de estudio 2003-2004 como variable independiente (en el eje $x$ ). La fórmula de cada línea fue calculada, así como el $\mathrm{R}^{2}$ de cada modelo de regresión.

\section{Resultados}

\subsection{Efectos de la herbivoría artificial y los niveles de luz en el crecimiento de las plántulas}

El crecimiento de plántulas es independiente del nivel de daño por herbivoría. Al utilizar la herbivoría natural como base de comparación en el análisis de covarianza se obtiene que no existe diferencia estadísticamente significativa en los efectos de dos tratamientos de herbivoría artificial (50\%, 95\%) sobre el crecimiento de las plántulas ( $>0.05$, Cuadro 1). Sin embargo, se observa una tendencia que a mayor daño foliar artificial, existe un menor crecimiento en altura de las plántulas. En el Cuadro 2, se observa que el nivel 2 de herbivoría artificial (50\%) reduce la media de altura promedio de $7.7 \mathrm{~cm}$ en $1.2 \mathrm{~cm}$ durante el periodo de estudio; mientras que el nivel 3 (95\% de herbivoría artificial) reduce la altura promedio en aproximadamente $1.9 \mathrm{~cm}$. La sobrevivencia de plántulas es independiente del nivel de daño causado por herbivoría foliar artificial $\left(\chi^{2}=0.323, \mathrm{gl}=2, \mathrm{P}=0.8508\right.$, Cuadro 2$)$. Al segregar los datos de sobrevivencia de plantas en el tratamiento de sombra y calcular nuevamente el Chi-cuadrado para esos datos, se encontró que la sobrevivencia de plántulas es independiente del nivel de daño causado por herbivoría artificial $\left(\chi^{2}=0.9622, \mathrm{gl}=2\right.$, $\mathrm{P}=0.9733)$.

El crecimiento de las plántulas en el experimento de intensidad de luz es estadísticamente dependiente de las alturas iniciales de las plántulas. Si se utiliza el tratamiento de sombra como la base de comparación, encontramos que el crecimiento de plántulas está negativamente relacionado con el tratamiento de intensificación de la luz (Cuadro 1). El resultado indica que la media del tamaño de las plántulas $(\mu \sim 7.2 \mathrm{~cm})$ se incrementa en $1 \mathrm{~cm}$ por año. El modelo presentado en la formula 1 representa una ANCOVA altamente significativa $\left(R^{2}=0.7457\right.$, $\mathrm{F}_{5,69}=50.59, \mathrm{p}=2.2 \mathrm{e}-16$, Cuadro 1 y 2). La Ilustración 1 muestra la altura de las 
plántulas en tiempo t como una función de la altura de plántulas en tiempo inicial t-1 y con códigos que representan tres tratamientos de intensidad de herbivoría artificial. El crecimiento de las plántulas muestra una tendencia a decrecer cuando los tratamientos de herbivoría fueron aplicados; sin embargo, esta relación no es estadísticamente significativa.

Cuadro 1. Resultados del Análisis de Covarianza (ANCOVA) para el modelo presentado en la fórmula 1

\begin{tabular}{cccccc}
\hline & Gl & $\begin{array}{c}\text { Suma } \\
\text { Cuadrados }\end{array}$ & $\begin{array}{c}\text { Media Suma } \\
\text { Cuadrados }\end{array}$ & $\mathrm{F}$ & $\operatorname{Pr}(>\mathrm{F})$ \\
\hline $\begin{array}{c}\text { Altura }\left(\mathrm{Y}_{\mathrm{t}-1}\right) \\
\text { Herbivoría }\end{array}$ & 1 & 15588.5 & 15588.5 & 196.5365 & $<2 \mathrm{e}-16^{* * *}$ \\
experimental $\left(H_{a}\right)$ & 2 & 89.6 & 44.8 & 0.5648 & 0.57105 \\
Luz $\left(L_{b}\right)$ & 2 & 371.3 & 371.3 & 4.6814 & $0.03396^{*}$ \\
Error $(\varepsilon)$ & 69 & 5472.8 & 79.3 & & \\
\hline Códigos de la significación estadística: $0^{\text {(***) }} 0.0010 .05^{\prime \prime} 1$ & &
\end{tabular}

Cuadro 2. Resultados de los coeficientes del ANCOVA presentados en la fórmula

1. Los parámetros están codificados en la primera columna y sus respectivos valores estimados en la segunda columna. El Error Estándar (E.S.) de cada parámetro, el valor de la prueba $t$ de los estimados y la probabilidad estadística de cada parámetro (p) se representan en las columnas que siguen.

\begin{tabular}{lllll}
\hline & Estimados & Error Std. & $\mathrm{t}$ & $\operatorname{Pr}(>|\mathrm{t}|)$ \\
\hline Media $(\mu)$ & 7.72572 & 3.65762 & 2.112 & $0.0383^{*}$ \\
Altura $\left(Y_{t-1}\right)$ & 0.91636 & 0.07219 & 12.694 & $<2 \mathrm{e}-16^{* * *}$ \\
$\mathrm{H}_{\mathrm{a}}(2)$ & -1.21437 & 2.48593 & -0.488 & 0.6267 \\
$\mathrm{H}_{\mathrm{a}}(3)$ & -1.86349 & 2.56206 & -0.727 & 0.4695 \\
$\mathrm{~L}_{\mathrm{b}}(2)$ & -4.73132 & 2.18671 & -2.164 & $0.0340^{*}$ \\
\hline
\end{tabular}

Códigos de la significación estadística: 0 “***) 0.05 '? 1

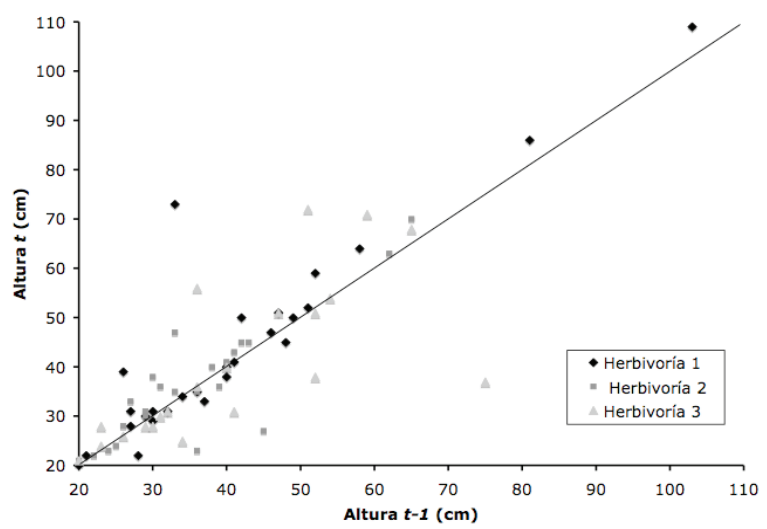

Ilustración 1. Relación entre tres niveles de herbivoría foliar y la altura de las plántulas de V. ferruginea 


\subsection{Efectos de la herbivoría natural sobre el crecimiento y la mortalidad de las plántulas}

El crecimiento de plántulas se correlaciona positivamente con el tamaño de las plántulas en el año anterior ( $<<0.001$, Cuadro 2). El modelo estadístico presentado en la formula 2 es una ANCOVA altamente significativo $\left(R^{2}=0.7872, F_{5,142}=105.1\right.$, $\mathrm{p}=2.2 \mathrm{e}-16$, Cuadro 3 y 4). La media del tamaño de las plántulas $\left(\mu^{\sim} 11.8 \mathrm{~cm}\right)$ se incrementa a razón de $86 \mathrm{~mm}$ por año. Además, el crecimiento de las plántulas es dependiente del nivel de daño por herbivoría natural. El resultado indica que la media de la altura de las plántulas $\left(\mu^{\sim} 11.8 \mathrm{~cm}\right)$ decrece $21 \mathrm{~mm}$ por cada $1 \%$ de herbivoría natural en 2003 ( $\mathrm{p}=0.05$, Cuadro 4), la altura incrementa en $29 \mathrm{~mm}$ por cada 1\% de herbivoría natural en 2004 ( $\mathrm{p}=0.05$, Cuadro 4). En presencia de arañas, la media de altura decrece $1.7 \mathrm{~cm}$ en 2003, e incrementa $3.1 \mathrm{~cm}$ en 2004, ambos resultados no fueron estadísticamente significativos. La Ilustración 2 muestra la relación entre la herbivoría en tiempo inicial $(t-1)$ y la suerte que corren las plántulas en tiempo t. Se encontró una diferencia estadística entre las medias de la herbivoría natural de las plántulas en tiempo t-1 cuando se comparan en relación con su estado (vivo contra muerto) en el siguiente periodo de medición $(t=-14.2457, \mathrm{df}=202, \mathrm{p}$ $<2.2 \mathrm{e}-16)$.

En la Ilustración 3 cada punto representa los valores individuales del porcentaje de herbivoría foliar como una función de la distancia al árbol congénere más cercano en el tiempo $(t-1, t)$. Los resultados indican que la distribución del nivel de daño por herbivoría es independiente de la distancia con respecto al adulto congénere más cercano en período $t-1\left(y=0.0194 x+9.5915 ; R^{2}=0.0001\right)$ y el periodo $t\left(y=0.1083+11.068 ; R^{2}=0.0039\right)$.

Cuadro 3. Resultados del análisis de covarianza (ANCOVA) para el modelo presentado en la fórmula 2

\begin{tabular}{|c|c|c|c|c|c|}
\hline & Gl & $\begin{array}{c}\text { Suma } \\
\text { Cuadrados }\end{array}$ & $\begin{array}{c}\text { Media Suma } \\
\text { Cuadrados }\end{array}$ & $\mathrm{F}$ & $\operatorname{Pr}(>F)$ \\
\hline Altura $\left(Z_{t: t}\right)$ & 1 & 99823 & 99823 & 518.8705 & $<2 \mathrm{e}-16^{* * *}$ \\
\hline Herbivoría ( $\mathrm{D}_{\mathrm{a}} 2003$ ) & 1 & 259 & 259 & 1.3471 & 0.24774 \\
\hline Herbivoría $\left(D_{a} 2004\right)$ & 1 & 650 & 650 & 3.3785 & 0.06814 \\
\hline Araña $04\left(A_{b}\right)$ & 1 & 315 & 315 & 1.6382 & 0.20267 \\
\hline Araña 03 ( & 1 & 36 & 36 & 0.1876 & 0.66557 \\
\hline Error $(\varepsilon)$ & 142 & 27319 & 191 & & \\
\hline
\end{tabular}

Cuadro 4. Resultados de los coeficientes del ANCOVA presentado en la fórmula 2. Los parámetros están codificados en la primera columna y sus respectivos valores estimados en la segunda columna. El Error Estándar (S.E.) de cada parámetro, el valor de la prueba $t$ de los estimados y la probabilidad estadística de cada parámetro (p) se muestran en las columnas que siguen. 


\begin{tabular}{lllll}
\hline & \multicolumn{1}{c}{ Estimados } & \multicolumn{1}{c}{ Error Std. } & \multicolumn{1}{c}{$\operatorname{Pr}(>|\mathbf{t}|)$} \\
\hline Altura $(\mu)$ & 11.80542 & 2.82399 & 4.180 & $5.06 \mathrm{e}-05^{* * *}$ \\
Altura $(2003)$ & 0.85602 & 0.03874 & 22.095 & $<2 \mathrm{e}-16^{* * *}$ \\
$\mathrm{D}_{\mathrm{a}}(2004)$ & 0.28916 & 0.15156 & 1.908 & 0.0584. \\
$\mathrm{D}_{\mathrm{a}}(2003)$ & -0.20705 & 0.11821 & -1.752 & 0.0820. \\
$\mathrm{~A} \mathrm{04}$ & 3.08738 & 2.34080 & 1.319 & 0.1893 \\
$\mathrm{~A} \mathrm{03}$ & -1.72930 & 3.99241 & -0.433 & 0.6656 \\
\hline \multicolumn{5}{c}{ Códigos de la significación estadística: $0^{\text {(***) }} 0.05 ! 11$}
\end{tabular}

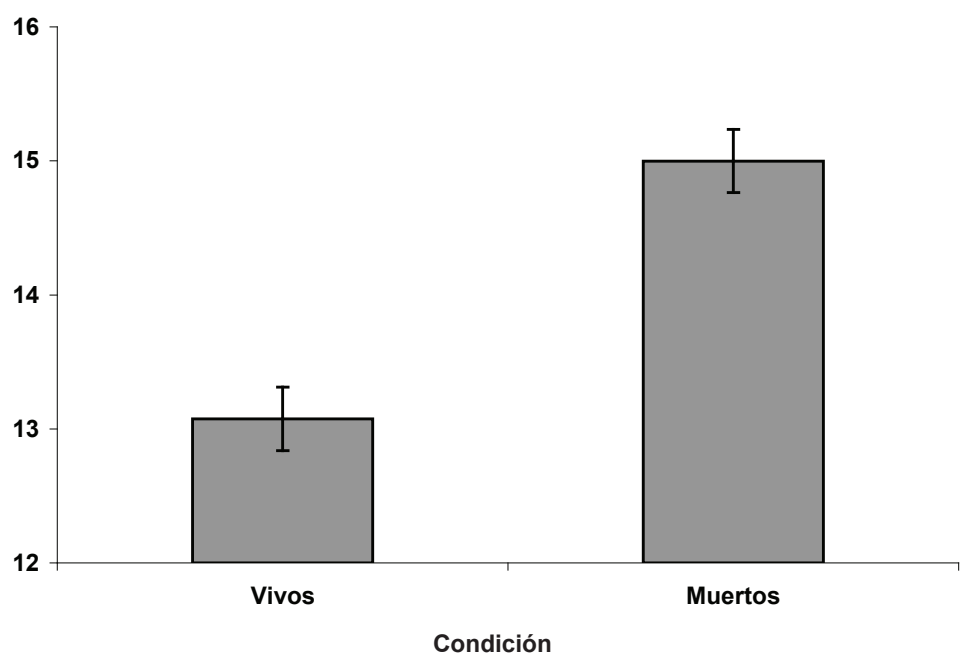

Ilustración 2. Condición de las plántulas en 2004 como una función de la herbivoría en febrero 2003 y las medias ( \pm E.S.), que fueron comparadas entre dos condiciones (vivos y muertos) en febrero 2004

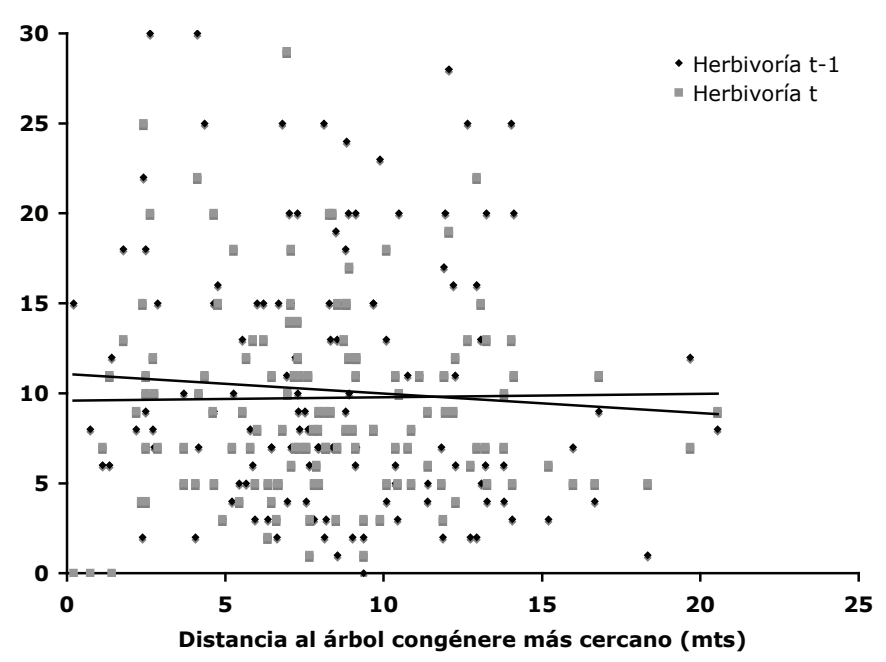

Ilustración 3. Porcentaje de la herbivoría foliar como una función de la distancia al árbol congénere más cercano de $V$. ferruginea $(\mathrm{m})$. Las líneas representan funciones de líneas rectas para dos períodos de medición: 2003 y 2004. 


\section{Discusión}

Se encontró una correlación positiva entre la altura inicial y el crecimiento de plántulas en un experimento y observaciones de campo. Los tratamientos de incremento en la infiltración de luz mostraron que existe correlación negativa entre las condiciones de mayor disponibilidad de luz y el crecimiento de las plántulas. El experimento de incremento de niveles de luz posiblemente causó la reducción en la humedad relativa del suelo y otros factores asociados, lo cual pudo haber afectado la fisiología de las plántulas y podría haber reducido el crecimiento de éstas. Tres niveles de herbivoría artificial no tuvieron efectos estadísticamente significativos en el crecimiento y la sobrevivencia de plántulas de V. ferruginea durante la primera etapa de estudio, algo también notado en otro experimento (Ruiz, 1997). Las plantas que sobrevivieron a la herbivoría pudieron haber compensado el daño causado por herbívoros mediante el uso de energía de reserva almacenada en raíces y tallos.

En estudios anteriores, con relación al efecto de la herbivoría foliar natural, se observó independencia entre crecimiento y mortalidad ante estos niveles de daño en V. ferruginea (Ruiz, 1997). El estudio anterior fue realizado en las parcelas de observaciones permanentes de nuestros sitios de estudio entre 1995 y 1997. Los resultados fueron sorprendentes puesto que se esperaba que porcentajes bajos de herbivoría ( 10\% de área foliar) tuvieran un efecto negativo y estadísticamente significativo sobre la sobreviviencia o el crecimiento de las plántulas. Los estudios recientes del efecto de la herbivoría foliar realizados en 2003-2004 proveen evidencia que sugiere un efecto negativo de la herbivoría. Inclusive cuando la herbivoría natural fue en promedio $\sim 10 \%$ del área foliar, este daño fue suficiente para reducir el crecimiento de las plántulas. Aparentemente, el cambio en el tiempo de la significancia de la herbivoría se relaciona con el porcentaje de cobertura en el dosel, producto del proceso de regeneración de bosques. En el estudio de la herbivoría natural realizado en 1996-1997 se argumentó que el efecto de la herbivoría podría ser considerable cuando el bosque alcanzara la etapa de raleo. En la etapa de raleo de bosques la competencia de las especies de árboles por sobrevivir en la copa crea condiciones de sombra en el sotobosque. En estas condiciones las plántulas podrían encontrarse en el punto de compensación de luz, donde la energía que aporta la fotosíntesis es equivalente a la energía utilizada en la respiración. En este punto de compensación las plántulas son vulnerables a la herbivoría puesto que la energía que se destina para defensa contra herbívoros es mínima. En estas condiciones, una porción de hoja consumida representa una sustracción significativa de la energía fotosintética necesaria para mantener las funciones vitales y para crecer. En síntesis, los resultados de las investigaciones en el bosque en raleo indican que la herbivoría natural es estadísticamente significativa y que tiene un efecto no lineal en el desarrollo del individuo, puesto que en un periodo la herbivoría se correlaciona con el crecimiento y en otro se correlaciona negativamente con éste. Las fluctuaciones del efecto de herbivoría natural en el crecimiento de las plántulas podría deberse a la presencia de arañas (Ruiz, Boucher, Ingram-Flores \& Chaves, 2009b) que alteran a la comunidad de insectos herbívoros. Esta última línea de estudio amerita mayor investigación. 
Se observó indicios de un efecto positivo indirecto entre la presencia de arañas y el crecimiento de plántulas de V. ferruginea. Aunque este efecto no es del todo estadísticamente significativo, algunas anotaciones de la historia natural y la ecología de esta interacción con la dinámica de bosques se presentan a continuación. Se observó que las arañas son depredadores naturales de insectos herbívoros, reducen por depredación el coeficiente de herbivoría de insectos herbívoros al posarse en las hojas de las plántulas - una interacción que amerita más investigación. He notado en el campo que las arañas acosan a los insectos y de esta forma seguramente reducen la herbivoría foliar en las plántulas de D. oleifera (Ruiz, Boucher, IngramFlores \& Chaves, 2009b). No obstante, la presencia de las arañas en las plántulas de $V$. ferruginea fue algo que no se observó en los primeros estudios de la regeneración de las plántulas de esta especie. Este efecto parece ser de naturaleza no lineal, puesto que varía en magnitud y dirección, probablemente de acuerdo al ciclo de los arácnidos. La abundancia y la composición de la comunidades de arañas y herbívoros foliares cambiaron durante el año. Sin embargo, no se cuenta con evidencia de que haya sido producto de depredación o por efecto de la época del año. En el otro estudio de campo realizado en nuestras parcelas de estudio se detectó un efecto positivo indirecto estadísticamente significativo de la presencia de araña sobre el crecimiento de las plántulas de Dipteryx oleifera (Ruiz, Boucher, Ingram-Flores \& Chaves, 2009b). En ambas investigaciones las arañas fueron principalmente constructoras de telas. Este tipo de arañas no reduce el área fotosintética porque construyen telas entre o debajo de las hojas y no entran en combate con las presas puesto que utilizan sus telas para capturarlas. Se ha encontrado una correlación positiva entre el comportamiento agresivo de morfo-especies de arañas y el área foliar total de plántulas de D. oleifera (Ruiz, Boucher, Ingram-Flores \& Chaves, 2009b). Las arañas en la familia Salticidae parecen ser eficientes depredadores en el sistema de $V$. ferruginea. Durante el desarrollo del experimento no se observaron herbívoros artrópodos en las plántulas de $V$. ferrugina, ni tampoco en las plántulas de D. oleifera en el otro estudio. Presumiblemente, el daño por herbivoría fue hecho durante la noche por insectos herbívoros nocturnos.

El nivel de daño de herbivoría es independiente de la distancia del árbol congénere más cercano en la especie Vochysia ferruginea. Este patrón sugiere la existencia de efectos ecológicos de insectos herbívoros generalistas, que consumen hojas de diversas especies (Janzen, 1970). En un estudio realizado en La Isla de Barro Colorado, cerca de $60 \%$ del daño causado a 9 especies de árboles fue por herbívoros especialistas (que consumen plantas en una misma familia) y el 8\% fue por generalistas, el resto del daño fue causado por patógenos (Coley \& Barone, 1996). En un estudio de la población de D. oleifera realizado de manera paralela, se observó que los niveles de herbivoría abarcaron ca. 10\% del area foliar de las plántulas. Los niveles de daño foliar son similares entre las dos especies en cuestión, aunque un poco más altos en $D$. oleifera. Una de las razones por las cuales la herbivoría es más alta en D. oleifera es la distribución agrupada de plántulas de esta especie (Ruiz, Boucher, Ruiz-Moreno \& Ingram-Flores, 2008). Esta distribución se relaciona con la presencia de daño de herbivoría cuando se encuentran en altas densidades (Janzen, 1970, Ruiz et al. 2009a). Este patrón concuerda con la función negativa observada entre la herbivoría y el individuo congénere más cercano en D. oleifera (Ruiz, Boucher, 
Ruiz-Moreno \& Ingram-Flores, 2009a), lo que indica que esta especie es afectada principalmente por insectos herbívoros especialistas. V. ferruginea tiene semillas muy pequeñas, dispersadas por el viento en patrones homogéneos de daño con respecto al árbol congénere más cercano. Este tipo de dispersión de semillas incrementa la eficiencia de ocupación de las plántulas por herbívoros generalistas en la especie.

En síntesis, el crecimiento de las plántulas depende de su altura. Los artrópodos herbívoros juegan un papel importante en la dinámica de las poblaciones de $V$. ferruginea. Este efecto cobra mayor importancia a medida que el bosque alcanza la fase de regeneración de raleo. El presente estudio es solamente correlativo y se necesita trabajar para establecer inequívocamente causas y efectos en las relaciones ecológicas entre las arañas, herbívoros artrópodos y la demografía de las plántulas. Independientemente de ésto, amerita investigación la idea propuesta: que las interacciones biológicas en cascadas tróficas cambian en magnitud y dirección durante el proceso de regeneración de bosques. Por ejemplo, incluir los efectos de las cascadas tróficas en las teorías de organización de bosques podría conducirnos a una mejor comprensión de cómo el reclutamiento de plántulas ocurre en los bosques húmedos tropicales.

\section{Agradecimientos}

La fase de campo de esta investigación fue posible gracias al apoyo financiero de la Fundación Nacional de Ciencias (BSR-8917688, DEB 9524061, DEB 0235761) a John Vandermeer; y el apoyo de la Fundación Nacional de Ciencias a instituciones de pregrado (DEB-0135350) a Douglas H. Boucher. Se agradece al Centro de Investigación y Documentación de la Costa Atlántica (CIDCA) por el fondo de becas de IDRC-Canadá para estudiantes de pregrado otorgado a Javier Ruiz. Para la fase de análisis de datos, redacción y publicación se utilizaron fondos de la International Foundation for Science (IFS; Estocolmo, Suecia) a partir de las becas de investigaciones a Javier Ruiz (D/3939-1; D/3939-2). La redacción del documento fue financiado por una Beca para Investigación IANAS 2012 otorgada a Javier Ruiz por las gestiones de la Academia Nicaragüense de Ciencias. Se agradece de forma especial a la Universidad de las Regiones Autónomas de la Costa Caribe Nicaragüense (URACCAN) y a la Bluefields Indian and Caribbean University (BICU) por su apoyo administrativo en la ejecución de las becas de IFS. De forma muy especial agradezco a los miembros del Proyecto Biodiversidad por su aporte en muchas formas.

\section{Referencias bibliográficas}

Augspurger, C. K. \& Kitajima, K. (1992). Experimental studies of seedling recruitment from contrasting seed distribution. Ecology, 73, 1270-1284.

Augspurger, C. K. (1983). Seed dispersal of the tropical tree, Platypodium elegans, and the escape of its seedlings from fungal pathogens. J. Ecol., 71, 759-771.

Augspurger, C. K. (1984). Seedling survival of tropical tree species: interactions of dispersal distance, light-gaps, and pathogens. Ecology, 65, 1705-1712. 
Basset, Y. (2001). Invertebrates in the canopy of tropical rain forests. How much do we really know? Plant Ecol., 153, 87-107.

Boucher, H. D. \& Granzow de la Cerda, I. (2008). Regeneración del dosel del bosque huracanado de La Bodega, Rio Kukra, R.A.A.S., a los dieciséis años del huracán Juana. Wani, (52), 17-21.

Brokaw, N. V. L. (1985). Gap-Phase Regeneration in a Tropical Forest. Ecology, 66, 682-687.

Carpenter, S. R., Cole, J. J., Hodgson, J. R., Kitchell, J. F., Pace, M. L., Bade, D., Cottingham, K. L., Essington, T. E., Houser, J. N. \& Schindler, D. E. (2001). Trophic cascades, nutrients, and lake productivity: whole-lake experiments. Ecol. Monog, 71, 163-186.

Clark, D. A. \& Clark, D. B. (1984). Spacing dynamics of a tropical rain forest tree: evaluation of the Janzen-Connel model. Am. Nat., 124, 769-788.

Clark, D. B. \& Clark, D. A. (1985). Seedling dynamics of a tropical tree: impacts of herbivory and meristem damage. Ecology, 66, 1884-1892.

Coley, D. \& Barone, J. (1996). Herbivory and plant defenses in tropical forest. Annu. Rev. Ecol. Syst., 27, 305-335.

Coley, P. D. (1983). Intraspecific variation in herbivory on two tropical tree species. Ecology, 64, 426-433.

Coley, P. D. (1990). Tasas de herbivorismo en diferentes árboles tropicales. En E.G. Leigh Jr., A. S. Rand \& D. M. Windsor (eds.). Ecología de un bosque tropical: Ciclos estacionales y cambios a largo plazo. (pp. 191-199). Panamá: Instituto Smithsonian de Investigaciones Tropicales.

Coley, P. D., Bryant, J. P. \& Chapin, F. S. I. (1985). Resource availability and plant antiherbivore defense. Science, 230, 895-899.

Dirzo, R. (1987). Estudio sobre interacciones planta herbívoros en Los "Tuxtlas" Veracruz. Rev. Biol. Trop., 35, 119-132.

Granzow-de la Cerda, I., Zamora, N., Vandermeer, J. H. \& Boucher, D. H. (1997). Biodiversidad de especies arbóreas en el bosque tropical húmedo del Caribe Nicaragüense siete años después del huracán Juana. Rev. Biol. Trop., 45, 1409. 1419.

Gruner, D. S. (2004). Attenuation of top-down and bottom-up forces in a complex terrestrial community. Ecology, 85, 3010-3022.

Hairston, N. G., Frederick, E. S. \& Lawerence, B. S. (1960). Community structure, population control, and competition. Am. Nat., 94, 421-425.

Hyatt, L. A., Rosenberg, M. S., Howard, T. G., Bole, G., Fang, W., Anastacia, K., Brown, K., Grella, R., Hinman, K., Kruidiziel, J. P. \& Gurevitch, J. (2003). The distance dependence prediction of the Janzen-Connell hypothesis: a meta-analysis. Oikos, 103, 590-602.

Janzen, D. H. (1970). Herbivores and the number of tree species in tropical forest. Am. Nat., 104, 501-528.

Levins, R. (1974). Qualitative analysis of partially specified systems. Ann. N. York Acad. Sci., 231, 123-138.

Moreau, G., Elveleigh, E. S., Lucarotti, C. J. \& Quiring, D. T. (2006). Ecosystem alteration modifies the relative strength of bottom-up and top-down forces in a herbivore population. J. An. Ecol. 75, 853-861. 
Romero, G. Q. \& Vasconcellos-Neto, J. (2004). Beneficial effects of flower-dwelling predators on their host plant. Ecology, 85, 446-457.

Ruiz, J. (1997). Respuestas en sobrevivencia y crecimiento de plántulas de 'Vochysia ferruginea' ante niveles y tipo de daño causado por herbivoría foliar. Tesis de Licenciatura no publicada, Universidad Centroamericana, Managua, Nicaragua.

Ruiz, J. (1999). Mortalidad, crecimiento y fenología de plántulas del palo de mayo (Vochysia ferruginea) expuestas a la herbivoría foliar en el bosque huracanado del Caribe Nicaragüense. Wani, (24), 34-47.

Ruiz, J., Boucher, D. H., Ruiz-Moreno, D. \& Ingram-Flores, C. L. (2008). Tropical Tree Species Recruitment in Space. Trabajo presentado en The Annual Meeting of the Ecological Society of America, Milwaukee, Wisconsin (paper).

Ruiz, J., Boucher, D. H., Ruiz-Moreno, D. \& Ingram-Flores, C. L. (2009a). Recruitment dynamics of the tropical rainforest tree Dipteryx oleifera (Fabaceae) in Eastern Nicaragua. Rev. Biol. Trop., 57, 321-338.

Ruiz, J., Boucher, D. H., Ingram-Flores, C. L. \& Chaves, L. F. (2009b). Beneficial effect of spider presence on seedling recruitment of the neotropical tree Dipteryx oleifera (Fabaceae). Rev. Biol. Trop., 57, 837-846.

Sullivan, J. J. (2003). Density-dependent shoot-borer herbivory increases the age of first reproduction and mortality of neotropical tree saplings. Oecologia, 136, 96-106.

Van Bael, S. A. \& Brawn, J. D. (2005). The direct and indirect effects of insectivory by birds in two contrasting Neotropical forest. Oecologia, 145, 658-668.

Van Bael, S. A., Brawn, J. D. \& Robinson, S. K. (2003). Birds defend trees from herbivores in neotropical forest canopy. PNAS, 100, 8304-8307.

Venables, W. N. \& Ripley, B. D. (2002). Modern Applied statistics with S. New York: Springer.

Yih, K., Boucher, D. H., Vandermeer, J. H. \& Zamora, N. (1991). Recovery of the rainforest of southeastern Nicaragua after destruction by hurricane Joan. Biotropica, 23, 106-113. 